\title{
Study of Tumor Growth under Hyperthermia Condition
}

\author{
Qing Zhu, ${ }^{1}$ Aili Zhang, ${ }^{1}$ Ping Liu, ${ }^{1,2}$ and Lisa X. Xu1, \\ ${ }^{1}$ School of Biomedical Engineering, Shanghai Jiao Tong University, Shanghai 200240, China \\ ${ }^{2}$ Med-X Research Institute, Shanghai Jiao Tong University, Shanghai 200240, China \\ Correspondence should be addressed to Aili Zhang, zhangaili@sjtu.edu.cn and Lisa X. Xu, lisaxu@sjtu.edu.cn
}

Received 2 May 2012; Revised 22 July 2012; Accepted 23 July 2012

Academic Editor: Scott Penfold

Copyright () 2012 Qing Zhu et al. This is an open access article distributed under the Creative Commons Attribution License, which permits unrestricted use, distribution, and reproduction in any medium, provided the original work is properly cited.

\begin{abstract}
The new concept of keeping primary tumor under control in situ to suppress distant foci sheds light on the treatment of metastatic tumor. Hyperthermia is considered as one of the means for controlling tumor growth. To simulate the tumor growth, a continuum mathematical model has been introduced. The newest understanding of the Warburg effect on the cellular metabolism and diffusion of the nutrients in the tissue has been taken into consideration. The numerical results are compared with the in vivo experimental data by fitting the tumor cell doubling time/tumor cell growth rate under different thermal conditions. Both the tumor growth curve and corresponding average glucose concentration have been predicted. The numerical results have quantitatively illustrated the controlling effect on tumor growth under hyperthermia condition in the initial stage.
\end{abstract}

\section{Introduction}

Cancer is the second major cause of human death in the world, and its mortality rate is growing every year [1]. Treatments include surgery, radiotherapy, chemotherapy, and gene therapy. Thermal therapy has also been intended to locally destroy tumor cells or enhance the body defense against tumor cells. However, recurrent rate of malignant tumor is still high [2], and the efficacy of the existing therapeutic means is yet to be improved. A new concept has been proposed recently that the primary tumor suppresses distal foci $[3,4]$. This sheds new light on tumor treatment. Keeping the primary tumor in situ but restricting its size might enable the host to impede the development of distal foci and progression of metastasis.

For tumor growth, there are three distinct stages: avascular, vascular, and metastatic/invade stage. Mathematical models have been developed to perform parametric studies on factors influencing tumor growth or to evaluate the outcome of tumor treatment modalities [5, 6]. Model-based numerical studies would enable one to extrapolate more spatial and temporal information from the experimental findings and to make predictions [7]. Laird [8] first found that the tumor growth data-fitted Gompertz function could be used to simulate the entire growth curve, which was defined as an empirical model. Hu and Ruan [9] studied the suppression effect of immunity system on tumor growth by merging the Gompertz function into a cellular automaton model. Other mathematical models based on certain biological assumptions have also been attempted to predict tumor growth curve using fundamental physics, such as mass/energy conservation. Greenspan [10] introduced surface tension into the diffusion model developed by Burton [11]. Tumor growth/inhibition factors [12, 13], cell adhesions $[14,15]$, angiogenesis $[16,17]$ and invasion $[18$, 19] were further considered to describe tumor growth at different stages.

Models focusing on the avascular stage [20-27] have been well studied and could be easily applied to in vitro experiment. Ward and King [23, 24] and Casciari et al. [28] proposed a continuum mathematical model focusing on how nutrients' concentration affects tumor growth. These models typically consist of reaction-diffusion equations. Forbes [29] further incorporated energy metabolism (ATP production rate) into the growth model. However, most of these models have not taken the Warburg effect into consideration, which fundamentally differentiates the tumor cell metabolism from that of the normal cells.

In 1930, Warburg (1930) proposed that tumor cells preferentially underwent glycolysis when consuming glucose 


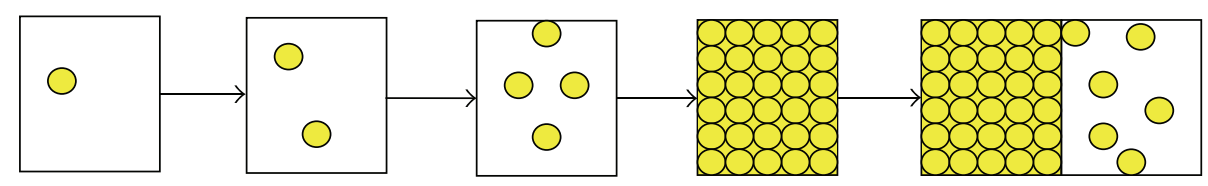

FIGURE 1: Schematic of tumor growth. The tumor starts with one single cell (represented by the yellow circle), and the cells differentiate continuously. When the infinitesimal unit of the space volume is filled up with the tumor cells, they grow into the next volume unit.

even under aerobic conditions. Unregulated glucose uptake and lactic acid production have been found in tumor cells as compared to normal cells $[30,31]$. It indicates that tumor cells obtain energy to maintain their viability primarily relying on anaerobic metabolism. This phenomenon was termed as "the Warburg effect." Anaerobic glycolysis consumes one molecule of glucose to produce 2 molecules of ATP as compared with oxidative phosphorylation which can produce 38 molecules of ATP [31-40]. Although the latter is much more efficient in glucose utilization, the rate of anaerobic glycolysis is much faster than aerobic metabolism. Therefore, the inefficient metabolism pathway might still supply enough energy for tumor cells to maintain their activities and differentiate at the cost of unreasonable consumption of glucose. The mechanisms causing the Warburg effect have been explained by gene mutation [38], signaling pathway alternations, possible defects in mitochondria [36, 41], and microenvironment deterioration (hypoxia or fluctuation of oxygen) [34, 37, 42]. Heiden et al. [32] have reported that biomass synthesis in tumor cells plays a role in the Warburg effect. Furthermore, he has determined nutrition utilizations in tumor cells: $85 \%$ of glucose converting to lactate in cytoplasm, 5\% reacting in mitochondria, and $10 \%$ synthesizing biomass. As the metabolic activities greatly influence the growth of tumor, it is necessary to include this unique metabolic mode of tumor in mathematical models.

Although thermal treatment has been applied in clinical applications for many years, most of them were used as short-term treatments. There are three classes of the treatment strategies [43-45]: with a mild temperature at $40 \sim 41^{\circ} \mathrm{C}$ for $6 \sim 72$ hours until the thermal dose is equivalent to 5 minutes at $43^{\circ} \mathrm{C}$; a moderate temperature at $42 \sim 45^{\circ} \mathrm{C}$ for $15 \sim 60$ minutes; and high temperature $>50^{\circ} \mathrm{C}$ for $4 \sim 6$ minutes. Both mild and moderate temperature treatments $\left(40 \sim 45^{\circ} \mathrm{C}\right)$ termed as hyperthermia treatment impair mammalian cells by protein denaturation and membrane damage and could cause cell death in situ [46].

In the present study, a mathematical model of tumor growth has been built by combining ATP production rate and the mechanism of the Warburg effect. It is validated by the tumor growth measurements in situ and further applied to study the hyperthermia effect on tumor metabolism over a period of time.

\section{Theory}

2.1. Experimental Study of Tumor Growth. Animal study of tumor metabolisms under long-term hyperthermia has been studied [47]. In the study, tumor cells were injected into the back of Balb/c mice around 6 8 weeks. Mice were grouped

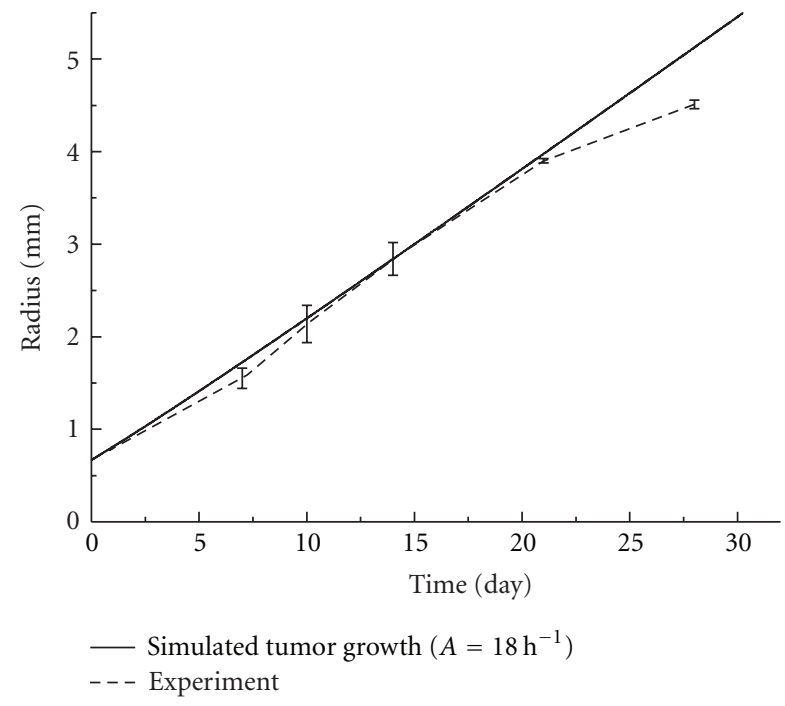

FIGURE 2: Tumor growth curve of the experimental measurements versus the numerical simulation of the control group.

randomly into the control and treatment groups with 6 in each group. In the treatment group, the tumor region of the mice was heated via a $15 \mathrm{~mm}$-diameter circular heating pad for 28 days. On each day, it was heated for 12 hours with an interval of 12 hours. The supplying power of the heating pad was controlled to maintain the tumor surface tissue temperature at $39^{\circ} \mathrm{C}$. Tumor sizes of both the control and treatment groups were measured and recorded at 3, 7, $10,14,21,28$ days, respectively. The experimental results are shown in Figures $2-5$ with the permission of the authors.

2.2. Model Development. At the avascular stage of a solid tumor, tumor cells proliferate and tumor grows like a spheroid without restriction. In our model, the tumor is assumed to start from one single tumor cell (as illustrated in Figure 1), and to grow into a homogenous spheroid over a period of time. This is an assumption used by many models $[7,10,14,16,23,24,29]$ and acceptable when the diameter of tumor is less than $2 \sim 4 \mathrm{~mm}$ prior to microvascular development. In addition, only living tumor cells are supposed to take the space of the spheroid. The proliferation of tumor cells is related to local concentration of nutrients. In this model, nutrients are simplified to the main source of energy (glucose) only. Glucose diffuses passively into tumor tissue from the outer rim of the tumor, where its concentration remains at a constant over the initial tumor growth period. 


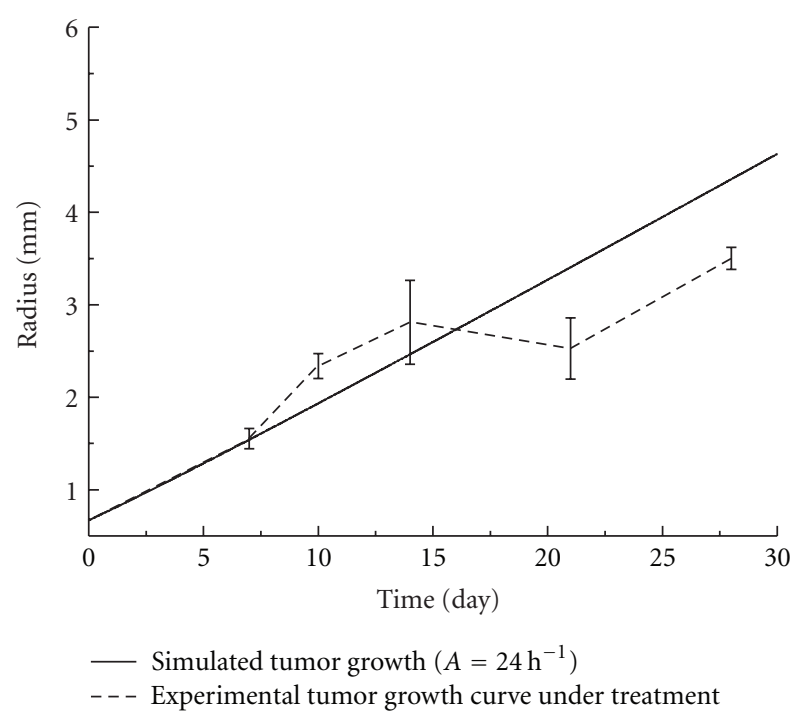

Figure 3: Tumor growth curve of the experimental measurements versus the numerical simulation of the treatment group $\left(39^{\circ} \mathrm{C}\right)(n=$ $6)$.

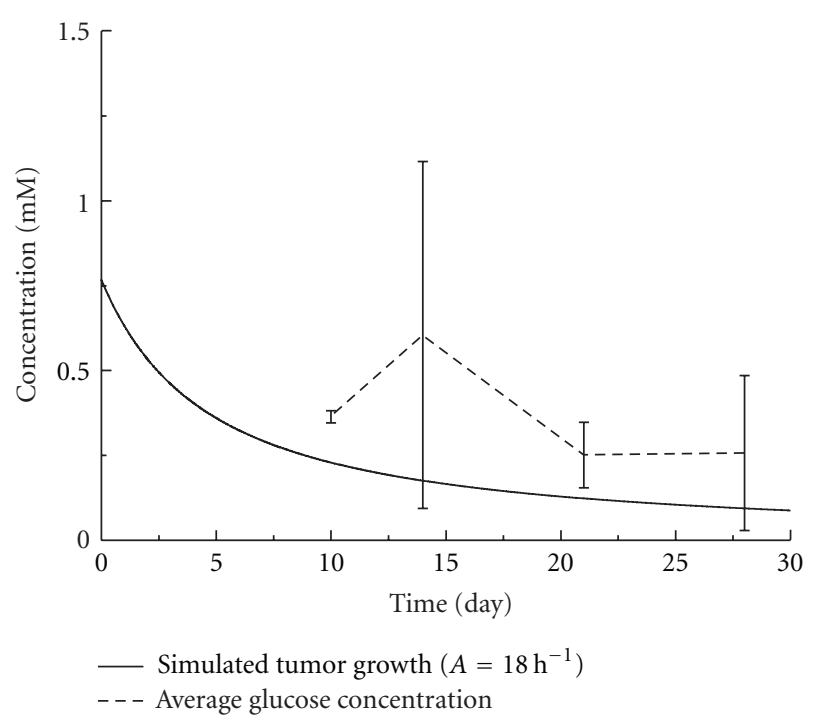

FIGURE 4: Average glucose concentration of the experimental measurements versus the numerical simulation of the control group.

Usually, the glucose is metabolized through two different pathways: aerobic and anaerobic, depending on the cell status and physiological conditions. According to the recent understanding of the Warburg effect $[32,38]$, in tumor metabolism, about $5 \%$ of glucose undergoes aerobic pathway, and $85 \%$ takes the anaerobic pathway to produce ATP. The rest of $10 \%$ glucose is utilized for biomass synthesis necessary for cell divisions.

Through anaerobic pathway, glucose first degrades into pyruvate, and pyruvate converts into lactate by lactate dehydrogenase in the cytoplasm. While in the aerobic metabolism, pyruvate will further react with oxygen, and produce water and carbon dioxide inside mitochondria. It is clear that

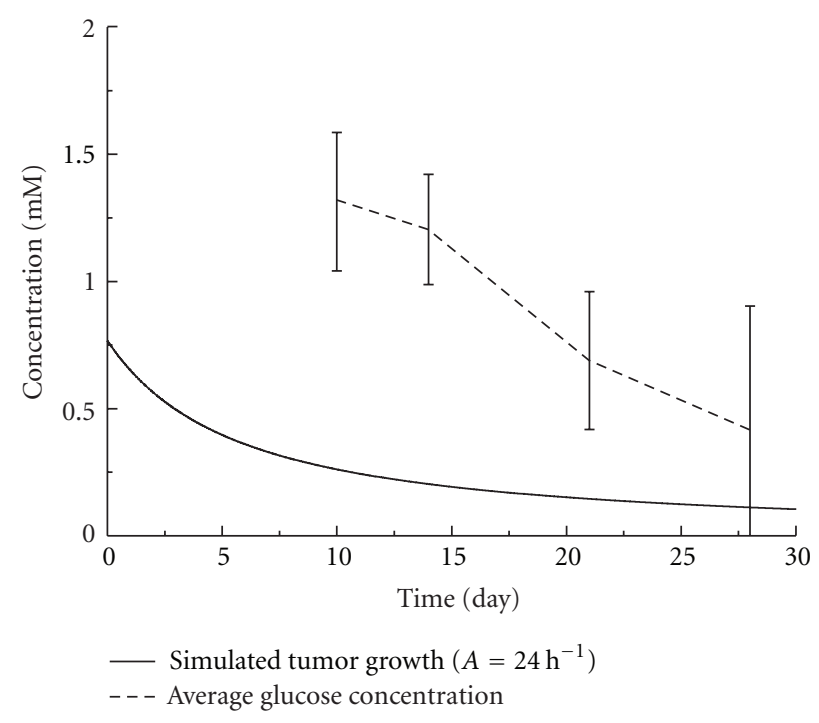

Figure 5: Average glucose concentration of the experimental measurement versus the numerical simulation of the treatment group.

not only the metabolic site and end products are different, but also the amount of energy produced differs. Through aerobic metabolism, one mole of glucose consumes 6 moles of oxygen and produces 38 moles of ATP, while in anaerobic metabolism only 2 moles of ATP per mole glucose could be produced without oxygen. These reactions are simplified and presented by the following formulae

Aerobic respiration:

$$
\mathrm{C}_{6} \mathrm{H}_{12} \mathrm{O}_{6}+6 \mathrm{O}_{2}+38 \mathrm{ADP} \longrightarrow 6 \mathrm{CO}_{2}+6 \mathrm{H}_{2} \mathrm{O}+38 \mathrm{ATP}
$$

Anaerobic respiration:

$$
\mathrm{C}_{6} \mathrm{H}_{12} \mathrm{O}_{6} \longrightarrow 2 \mathrm{C}_{3} \mathrm{H}_{6} \mathrm{O}_{3}+2 \mathrm{ATP}
$$

The proliferation rate of tumor cells is supposed to rely on the ATP production rate, which is determined by the metabolic reactions:

$$
Q_{\mathrm{ATP}}=38 Q_{g, \mathrm{AR}}+Q_{\mathrm{lac}},
$$

where $Q_{g, A R}$ is the glucose assumption rate due to aerobic respiration, $Q_{\text {lac }}$ is the lactate production rate.

Following the hypothesis of Heiden [32], the glucose consumption rate due to aerobic metabolism and the lactate production rate could be derived from the uptake rate of glucose by tumor cells:

$$
\begin{gathered}
Q_{g, \mathrm{AR}}=5 \% Q_{g}, \\
Q_{\text {lac }}=85 \% \times 2 \times Q_{g},
\end{gathered}
$$

where $Q_{g}$ is the cellular glucose uptake rate.

Then the ATP production rate $Q_{\text {ATP }}$ could be calculated:

$$
Q_{\text {ATP }}=5 \% \times 38 \times Q_{g}+85 \% \times 2 \times Q_{g} .
$$


The cellular uptake rate of glucose depends on both the extracellular level of glucose concentration and the ability of cell uptake. The reaction is assumed to be enzyme reaction and followed Michaelis-Menten kinetics' equation [10, 25]:

$$
Q_{g}=Q_{g \max } \frac{c_{g}}{c_{g}+K_{g}},
$$

where $Q_{g \max }$ is the maximum glucose uptake rate per tumor cell, $c_{g}$ is the glucose concentration in tumor tissue, and $K_{g}$ is saturate concentration.

The concentrations of nutrients (both glucose and lactate) are assumed to be functions of tumor spheroid radius that are changing with the rate of cell proliferation [7]:

$$
\frac{d c_{g}}{d t}=D_{g} \nabla^{2} c_{g}-Q_{g} l
$$

where $D_{g}$ is the diffusion coefficient of glucose and reported to be $1.05 * 10^{-6} \mathrm{~cm}^{2} / \mathrm{s}$ for EMT6/R0 spheroid, and $l$ is the number of living cell per unit volume.

The concentration of glucose at the boundary is decided by the average value of glucose concentration in blood$5 \mathrm{mM}$, and the tumor is assumed symmetric, thus the boundary conditions for (5) are as follows

Boundary condition:

$$
\left.\frac{\partial c_{g}}{\partial t}\right|_{r=0}=0,\left.\quad c_{g}\right|_{r=R}=5 \mathrm{mM}
$$

where $R$ is the radius of solid tumor.

The distribution of lactate in tumor is determined by

$$
\frac{d c_{\mathrm{lac}}}{d t}=D_{\mathrm{lac}} \nabla^{2} c_{\mathrm{lac}}-Q_{\mathrm{lac}} l,
$$

where $c_{\text {lac }}$ is the concentration of lactate in tumor tissue, and $D_{\text {lac }}$ is the effective diffusion coefficient of glucose and lactate. The boundary conditions are:

$$
\left.\frac{\partial c_{\mathrm{lac}}}{\partial t}\right|_{r=0}=0,\left.\quad c_{\mathrm{lac}}\right|_{r=R}=0 \mathrm{mM}
$$

The diffusion coefficient of lactate could be determined from the glucose diffusivity [25]:

$$
D_{\mathrm{lac}}=D_{g}\left(\frac{M W_{g}}{M W_{\mathrm{lac}}}\right)^{3 / 4},
$$

where $M W_{g}$ and $M W_{\text {lac }}$ are molecular weights of glucose and lactate, respectively.

Assuming that all viable space in tumor tissue is filled with living and tightly packed tumor cells (see Figure 1), the number of living cells per unit tumor tissue volume is

$$
l=\frac{1}{V_{L}},
$$

where $V_{L}$ is the volume of a living tumor cell. It is obtained through in vitro measurement. Diameters of more than 150 suspended 4T1 tumor cells are measured and averaged to get the volume of a single cell. Its average radius is $13.41 \mu \mathrm{m}$ with a standard deviation of $2.21 \mu \mathrm{m}$. With this radius, the tumor cell volume was calculated and the result is listed in Table 1.

By solving the above listed equations together, the ATP production rate and concentrations of glucose and lactate inside the tumor tissue could be obtained. The relationship between ATP production rate and cell growth rate is proposed and well parameterized by Forbes et al. [29]:

$$
k_{l}=A \frac{Q_{\text {ATP }}}{Q_{\text {ATP }}+K_{\text {ATP }}},
$$

where $k_{l}$ is cell growth rate of tumor cells, $A$ is the maximum cell growth rate, and $K_{\mathrm{ATP}}$ is saturation ATP production rate.

In this model, tumor growth is considered as tumor cells moving outward while all space occupied. In other words, tumor growth is mainly due to living tumor cells' proliferation, then the moving velocity of the tumor spheroid rim is given as

$$
v\left(t, R_{(t)}\right)=\frac{\int_{0}^{R_{(t)}} k_{l} l V_{L} r^{2} d r}{R_{(t)}^{2}},
$$

where $r$ is radial distant from center of tumor spheroid, and $R_{(t)}$ is the radius of tumor spheroid at time $t$. The radius of tumor spheroid at time $t+d t$ is

$$
R_{(t+d t)}=\int_{t}^{t+d t} v\left(t, R_{(t)}\right) d t+R_{(t)}
$$

The average concentration of the metabolites is calculated from the integration of the substances' distribution throughout tumor tissue divided by the tumor tissue volume as

$$
\bar{c}_{(t)}=\frac{\int_{0}^{R_{(t)}}\left(c(r) \cdot 4 \pi r^{2}\right) d r}{(4 / 3) \pi R_{(t)}^{3}} .
$$

By substituting the dimensionless quantities and parameters as defined below into the above equations, the dimensionless equations could be obtained.

The dimensionless variables are defined as

$$
c_{g}^{*}=\frac{c_{g}}{c_{g, 0}}, \quad c_{\mathrm{lac}}^{*}=\frac{c_{\mathrm{lac}}}{c_{g, 0}}, \quad r^{*}=\frac{r}{R_{(t)}^{*}}, \quad t^{*}=\frac{t}{1 / A},
$$

where $c_{g, 0}=5 \mathrm{mM}$. 
TABLE 1: List of variables used in modeling.

\begin{tabular}{lcc}
\hline Variable & Description & Unit \\
\hline$c_{g}$ & Glucose concentration & $\mathrm{mM}$ \\
$c_{\text {lac }}$ & Lactate concentration & $\mathrm{mM}$ \\
$Q_{g}$ & Glucose uptake rate & $\mathrm{mol} / \mathrm{cell} / \mathrm{sec}$ \\
$Q_{\text {lac }}$ & Lactate production rate & $\mathrm{mol} / \mathrm{cell} / \mathrm{sec}$ \\
$Q_{\text {ATP }}$ & ATP production rate & $\mathrm{mol} / \mathrm{cell} / \mathrm{sec}$ \\
$k_{l}$ & Cell proliferation time & $1 / \mathrm{h}$ \\
$L$ & Number of living cells in certain unit volume & $\mathrm{cells} / \mathrm{cm}^{3}$ \\
$R$ & Radius of tumor spheroid & $\mathrm{cm}$ \\
$V$ & Tumor growth velocity & $\mathrm{cm} / \mathrm{h}$ \\
$T$ & Growth time & $\mathrm{h}$ \\
\hline
\end{tabular}

Thus, the dimensionless equations are

$$
\begin{gathered}
Q_{\mathrm{ATP}}^{*}=85 \% * 2 * Q_{g}^{*}+5 \% * 38 * Q_{g}^{*} \\
Q_{g}^{*}=\frac{Q_{g}}{Q_{g \max }}=\frac{c_{g}^{*}}{c_{g}^{*}+K_{g} / c_{g, 0}} \\
Q_{\mathrm{lac}}^{*}=\frac{Q_{\mathrm{lac}}}{Q_{g \max }}=\frac{c_{\mathrm{lac}}^{*}}{c_{\mathrm{lac}}^{*}+K_{\mathrm{lac}} / c_{g, 0}} \\
\frac{d c_{g}^{*}}{d t^{*}}=\frac{1}{A}\left(\nabla^{2} c_{g}^{*}-\frac{R(t)^{2} Q_{g \max }}{D V_{L} c_{g, 0}} Q_{g}^{*}\right) \\
\left.\frac{d c_{\mathrm{lac}}^{*}}{d t^{*}}=\left.\frac{1}{A}\right|_{r^{*}=1}=1 \nabla^{2} c_{\mathrm{lac}}^{*}-\frac{R(t)^{2} Q_{g \max }}{D V_{L} c_{g, 0}} Q_{\mathrm{lac}}^{*}\right) \\
\left.c_{\mathrm{lac}}^{*}\right|_{r^{*}=1}=0 \quad \frac{\left.d c_{\mathrm{lac}}^{*}\right|_{r^{*}=0}}{d t^{*}}=0 \\
k_{l}^{*}=\frac{k_{l}}{A}=\frac{Q_{\mathrm{ATP}}^{*}}{Q_{\mathrm{ATP}}^{*}+K_{\mathrm{ATP}} / Q_{g} \max } \\
R_{(t)}=\int_{0}^{t}\left(v^{*} R_{(t)} A\right) d\left(\frac{t^{*}}{1 / A}\right)+R_{0} .
\end{gathered}
$$

Tumor growth in vivo is actually a complex process, which involves many influencing factors such as gene mutation, immune system, tumor cell mechanism effect, tumor angiogenesis, metabolic waste, and tumor microenvironment. It is difficult to include all these factors into one single model. The present model is built based on energy production only and all other influencing factors are lumped into the maximum cell growth rate parameter $A$. The behavior of tumor growth is determined by the maximum cell growth rate $A$ and it is determined by fitting to the animal experiment data. The other parameters used are all listed in Tables 1 and 2. The constants $\left(D_{g}, Q_{g} \max \right)$ listed in Table 2 are taken from EMT6/Ro tumor, whose biophysical constants have been well studied. EMT6 is a mouse breast cancer cell line that grows in the Balb/C strain. 4T1 cells are assumed to be similar to EMT6 biophysically in the present modeling.
To solve the equations listed above, discrete algorithm is built. The one-dimensional tumor space is divided into 1000 intervals evenly. The time increment $\Delta t$ is set to be small enough to guarantee the solver stability. The diffusionreaction equations are then differentiated using the finite difference method. The tumor radius at certain time $t$ is used as an input into the differentiated equation, and the forward elimination and backward substitution method is used to solve these differentiated equations to obtain the glucose concentration distribution, the corresponding ATP production rate, tumor growth rate, the velocity of the moving boundary of tumor, and the new tumor radius at a given time $t$. The updated tumor radius is then used as an input into the equations iteratively, until the difference between two iterations is small enough. For the next time step $(t+\Delta t)$, the radius of tumor is derived from the original radius at time $t$ and radius increment during $\Delta t$. This process is repeated until the final convergence reached in simulation.

The maximum cell growth rate parameter $A$ is fitted from the animal experimental data. The reciprocal of $A$ ranged from $0 \sim 50 \mathrm{~h}$, with an interval of $0.2 \mathrm{~h}$. Each $A$ is then substituted to the mathematical model to obtain a simulated curve, which is compared with the animal experimental data using the coefficient of determination (R2) as a criteria. The optimal $A$ is chosen in correspondance to the maximum achieved $\mathrm{R} 2$ value.

\section{Results and Discussion}

By fitting the numerical model with the experimentally measured tumor growth data, the parameter $A$ for tumor growth with and without treatment was obtained. The fitted curve and the experimental results were shown in Figures 2 and 3 , respectively.

For the control group, the tumor growth curve during the first 28 days was well captured by the energy-based model developed in this study (Figure 2). The fitted maximum cell growth rate parameter $A$ was $(18 \mathrm{~h})^{-1}$. However, after 28 days, the tumor growth slowed down and became nonlinear, which could no longer be described by the current mathematical model. It implied that alternations in tumor metabolism and some impedimental mechanisms might appear which led to cell death when tumor radius 
TABLE 2: List of constants used in modeling (cell type-EMT6/Ro).

\begin{tabular}{lccc}
\hline Variable & Description & Value & Authors \\
\hline$D_{g}$ & Glucose diffusion coefficient & $1.05 * 10^{-6} \mathrm{~cm}^{2} / \mathrm{s}$ & Casciari et al., 1988 [28] \\
$Q_{g \max }$ & Maximum glucose uptake rate & $1.33 * 10^{-16} \mathrm{~mol} /$ (cell sec) & Casciari et al., 1992 [25] \\
$K_{g}$ & Glucose saturation constant & $4.0 * 10^{-2} \mathrm{mM}$ & Casciari et al., 1992 [25] \\
$V_{L}$ & Living cell volume & $1.26 * 10^{-9} \mathrm{~cm}^{3}$ & Experiment \\
$c_{g, 0}$ & Glucose concentration at tumor rim & $5.5 \mathrm{mM} \mathrm{or} \mathrm{less}$ & Freyer and Sutherland, 1986 [55] \\
$K_{\mathrm{ATP}}$ & Cell growth saturation constant & $3.75 * 10^{-19} \mathrm{~mol} / \mathrm{cells}$ & Venkatasubramanian et al., 2006 [29] \\
\hline
\end{tabular}

reached about $4 \sim 5 \mathrm{~mm}$ in diameter. These factors were not considered in the present model.

Tumor growth in mice under long-term mild hyperthermia treatment was also fitted and shown in Figure 3. It was obvious that the growth rate of tumor was much slower and the fitted maximum cell growth rate $A$ was $(24 \mathrm{~h})^{-1}$. The model predicted the general trend of tumor growth well in the first 15 days. The oscillation occurred in the growth curve that was not observed in the control group could be attributed to the thermal interference, although the causing mechanism is yet to be further explored. Moreover, it was clear that the model overestimated the tumor growth under the hyperthermia condition after 15 days. This indicates that more complex effects on the system might be triggered by hyperthermia after a period of time. It could be related to tumor angiogenesis, the activities of enzymes in different pathways associated with tumor cell metabolism and proliferation and so forth, which cannot be simply modeled based on energy consumption.

With the fitted parameter $A$, the distribution of glucose in tumor has also been obtained. The average concentration of glucose in the tumor was calculated and compared to the experimental results. Seen from Figures 4 and 5, the average concentrations of glucose tended to decrease which agreed well with the experimental results. There existed significant differences between experimental and numerical results, especially for the group under the long-term mild hyperthermia treatment. This was likely because in the present model only glucose consumption for cell proliferation was considered. There could be excessive supply of glucose from the conceivable blood vessels inside tumor and the decreased cellular uptake of glucose due to heat. A more sophisticated model taking these factors into consideration should be developed to accurately predict the glucose distribution in tumor tissue.

As there are no publications on the nutrition diffusion and cell uptake rate of 4T1 tumor cells, the data for EMT6/Ro tumor has been used. Although EMT6/Ro tumor cells are expected to possess similar properties, the dependence of these parameters on tumor species might also introduce some errors in the simulations. Therefore, parametric studies were performed and the influences on the glucose uptake rate and the diffusion coefficient were studied as shown in Figures 6 and 7. It was clear that the variation of tumor growth caused by $10 \%$ changes of either two factors was less than $5 \%$.

In the present model, besides the Warburg effect, the influence of all other factors such as blood perfusion,

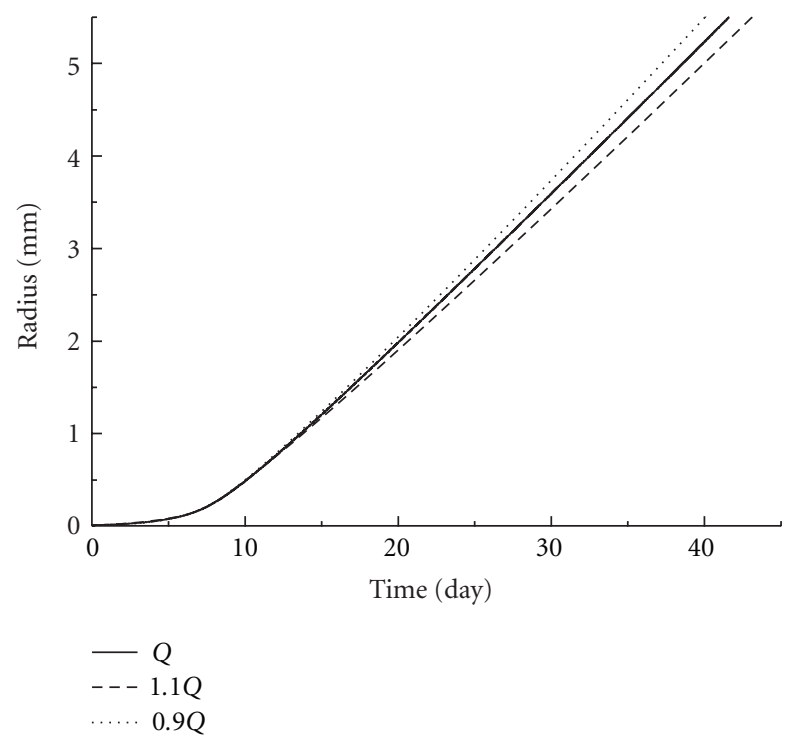

FIGURE 6: Parametric study of the tumor growth rate under different glucose uptake rates in tumor cells. Solid line stands for glucose uptake rate from reference [29]. Dot and dash lines represent 10\% increase and decrease, respectively.

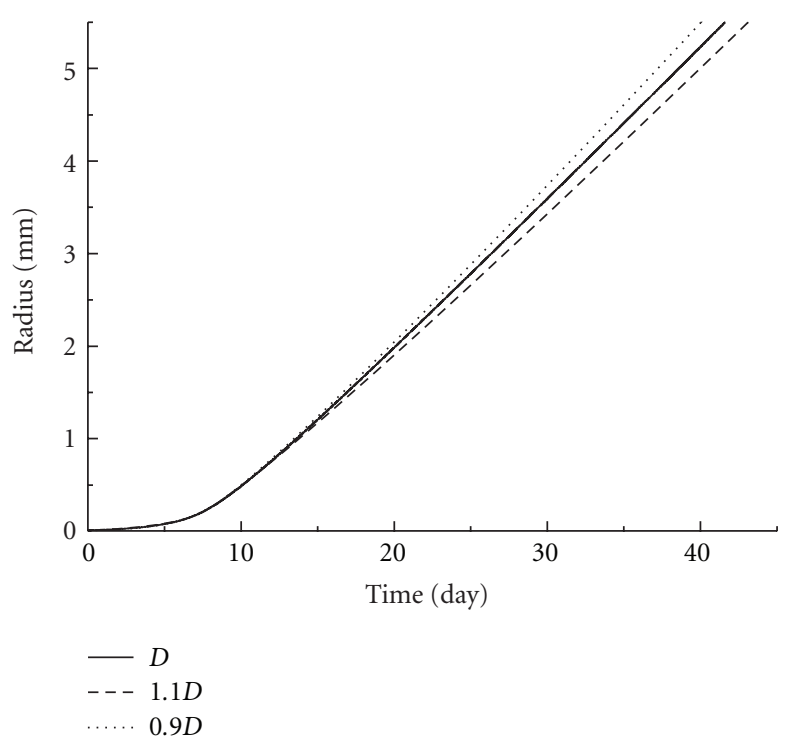

Figure 7: Parametric study of the tumor growth rate under different glucose diffusion coefficients in tumor tissue. Solid line stands for glucose diffusion value from reference [29]. Dot and dash lines represent $10 \%$ increase and decrease, respectively. 
immunity, and other growth or necrosis factors, has been lumped into a simple parameter $A$. Using tumor growth data from the experimental studies, the maximum tumor cell growth rate in vivo under the normal condition was fitted to be $(18 \mathrm{~h})^{-1}$, and the linear tumor growth at the early stage was successfully modeled without consideration of cell death. The growth was found to be excessively inhibited by the long-term mild hyperthermia treatment. In fact, during the treatment, both the temperature gradient and inhomogeneity existed, and the higher temperature could impair tumor cells by denaturation or destruction of cellular membrane, cellular skeleton, and nucleus [48]. The long-term mild hyperthermia treatment might also affect the activities of enzymes in different pathways associated with tumor cell metabolism and proliferation, stimulate the immunology factors such as hsp70, which could arouse body system defense and eliminate tumor cells specifically [49]. Besides, Song's study [50] revealed that under mild hyperthermia $\left(41 \sim 42^{\circ} \mathrm{C}, 30 \mathrm{~min}\right)$ blood perfusion in tumor tissue could increase 1.5 2 folds as compared to that prior to the treatment. Tumor vasculature is very sensitive to heat, as it is loosely organized and usually lacks of smooth muscles [51-54]. Tumor cells would suffer from starvation due to the damage of the angiogenesis. All these influences on tumor growth are not linear and could not be accurately represented by a single parameter. This warrants further investigation into more detailed modeling of the long-term hyperthermia effect on tumor growth in the near future.

\section{Conclusion}

An energy-based model linking tumor growth with cell proliferation rate has been developed in this study to investigate the hyperthermia treatment effect. In the model, the new understanding of the Warburg Effect was for the first time taken into account for tumor cellular metabolism regardless of the concentration of oxygen. The maximum cell growth rate was used as an integrated variable responding to changes under different environments. Trends of initial tumor growth and changes of the average glucose concentration in tumor were successfully modeled. The comparison of the maximum tumor cell growth rate has revealed a slowdown of tumor growth under the long-term mild hyperthermia condition. To accurately predict the tissue glucose level and the corresponding metabolites, especially under the longterm hyperthermia, the model needs to be further developed.

\section{Acknowledgments}

This work has been supported by the National Natural Science Foundation of China (NSFC51076095, NSFC50725622) and Shanghai Municipal Science and Technology Commission (10QA1403900).

\section{References}

[1] A. Jemal, R. Siegel, E. Ward, Y. Hao, J. Xu, and M. J. Thun, "Cancer statistics, 2009," CA Cancer Journal for Clinicians, vol. 59, no. 4, pp. 225-249, 2009.
[2] O. Abe, R. Abe, K. Enomoto et al., "Effects of radiotherapy and of differences in the extent of surgery for early breast cancer on local recurrence and 15-year survival: an overview of the randomised trials," Lancet, vol. 366, no. 9503, pp. 2087-2106, 2005.

[3] B. Fisher, N. Gunduz, and E. A. Saffer, "Influence of the interval between primary tumor removal and chemotherapy on kinetics and growth of metastases," Cancer Research, vol. 43, no. 4, pp. 1488-1492, 1983.

[4] K. Camphausen, M. A. Moses, W. D. Beecken, M. K. Khan, J. Folkman, and M. S. O'Reilly, "Radiation therapy to a primary tumor accelerates metastatic growth in mice," Cancer Research, vol. 61, no. 5, pp. 2207-2211, 2001.

[5] N. L. Komarova, "Mathematical modeling of tumorigenesis: mission possible," Current Opinion in Oncology, vol. 17, no. 1, pp. 39-43, 2005.

[6] T. W. Secomb, D. A. Beard, J. C. Frisbee, N. P. Smith, and A. R. Pries, "The role of theoretical modeling in microcirculation research," Microcirculation, vol. 15, no. 8, pp. 693-698, 2008.

[7] T. Roose, S. J. Chapman, and P. K. Maini, "Mathematical models of avascular tumor growth," SIAM Review, vol. 49, no. 2, pp. 179-208, 2007.

[8] A. K. Laird, "Dynamics of tumor growth," British journal of cancer, vol. 13, pp. 490-502, 1964.

[9] R. C. Hu and X. G. Ruan, "A simple cellular automaton model for tumor-immunity system," in Proceedings of the IEEE International Conference on Robotics, Intelligent Systems and Signal Processing, vol. 1-2, pp. 1031-1035, 2003.

[10] H. P. Greenspan, "Models for growth of a solid tumor by diffusion," Applied Mathematics, vol. 51, no. 4, pp. 317-340, 1972.

[11] A. C. Burton, "Rate of growth of solid tumours as a problem of diffusion," Growth, Development and Aging, vol. 30, no. 2, pp. 157-176, 1966.

[12] Y. Kim and A. Friedman, "Interaction of tumor with its microenvironment: a mathematical model," Bulletin of Mathematical Biology, vol. 72, no. 5, pp. 1029-1068, 2010.

[13] H. M. Byrne and M. A. Chaplain, "Growth of nonnecrotic tumors in the presence and absence of inhibitors," Mathematical Biosciences, vol. 130, no. 2, pp. 151-181, 1995.

[14] A. R. A. Anderson, "A hybrid mathematical model of solid tumour invasion: the importance of cell adhesion," Mathematical Medicine and Biology, vol. 22, no. 2, pp. 163-186, 2005.

[15] L. Preziosi and A. Tosin, "Multiphase modelling of tumour growth and extracellular matrix interaction: mathematical tools and applications," Journal of Mathematical Biology, vol. 58, no. 4-5, pp. 625-656, 2009.

[16] A. R. A. Anderson and M. A. J. Chaplain, "Continuous and discrete mathematical models of tumor-induced angiogenesis," Bulletin of Mathematical Biology, vol. 60, no. 5, pp. 857-899, 1998.

[17] P. Macklin, S. McDougall, A. R. A. Anderson, M. A. J. Chaplain, V. Cristini, and J. Lowengrub, "Multiscale modelling and nonlinear simulation of vascular tumour growth," Journal of Mathematical Biology, vol. 58, no. 4-5, pp. 765-798, 2009.

[18] J. A. Adam, "Mathematical models of prevascular spheroid development and catastrophe-theoretic description of rapid metastatic growth/tumor remission," Invasion and Metastasis, vol. 16, no. 4-5, pp. 247-267, 1996.

[19] A. M. Stein, T. Demuth, D. Mobley, M. Berens, and L. M. Sander, "A mathematical model of glioblastoma tumor spheroid invasion in a three-dimensional in vitro experiment," Biophysical Journal, vol. 92, no. 1, pp. 356-365, 2007. 
[20] D. Grecu, A. S. Carstea, A. T. Grecu et al., "Mathematical modelling of tumor growth," Romanian Reports in Physics, vol. 59, no. 2, pp. 447-455, 2007.

[21] Y. Jiang, J. Pjesivac-Grbovic, C. Cantrell, and J. P. Freyer, "A multiscale model for avascular tumor growth," Biophysical Journal, vol. 89, no. 6, pp. 3884-3894, 2005.

[22] J. A. Sherratt and M. A. J. Chaplain, "A new mathematical model for avascular tumour growth," Journal of Mathematical Biology, vol. 43, no. 4, pp. 291-312, 2001.

[23] J. P. Ward and J. R. King, "Mathematical modelling of avascular-tumour growth," IMA Journal of Mathemathics Applied in Medicine and Biology, vol. 14, no. 1, pp. 39-69, 1997.

[24] J. P. Ward and J. R. King, "Mathematical modelling of avascular-tumour growth II: modelling growth saturation," IMA Journal of Mathemathics Applied in Medicine and Biology, vol. 16, no. 2, pp. 171-211, 1999.

[25] J. J. Casciari, S. V. Sotirchos, and R. M. Sutherland, "Mathematical modelling of microenvironment and growth in EMT6/Ro multicellular tumour spheroids," Cell Proliferation, vol. 25, no. 1, pp. 1-22, 1992.

[26] M. A. J. Chaplain and B. D. Sleeman, "A mathematical model for the growth and classification of a solid tumor: a new approach via nonlinear elasticity theory using strain-energy functions," Mathematical Biosciences, vol. 111, no. 2, pp. 169215, 1992.

[27] M. A. J. Chaplain, "Avascular growth, angiogenesis and vascular growth in solid tumours: the mathematical modelling of the stages of tumour development," Mathematical and Computer Modelling, vol. 23, no. 6, pp. 47-87, 1996.

[28] J. J. Casciari, S. V. Sotirchos, and R. M. Sutherland, "Glucose diffusivity in multicellular tumor spheroids," Cancer Research, vol. 48, no. 14, pp. 3905-3909, 1988.

[29] R. Venkatasubramanian, M. A. Henson, and N. S. Forbes, "Incorporating energy metabolism into a growth model of multicellular tumor spheroids," Journal of Theoretical Biology, vol. 242, no. 2, pp. 440-453, 2006.

[30] O. Waruburg, "On the origin of cancer cells," Science, vol. 123, pp. 309-314, 1956.

[31] O. Waruburg, The Prime Cause and Prevention of Cancer, Konrad Triltsch, Würzburg, Germany, 1969.

[32] M. G. V. Heiden, L. C. Cantley, and C. B. Thompson, "Understanding the warburg effect: the metabolic requirements of cell proliferation," Science, vol. 324, no. 5930, pp. 1029-1033, 2009.

[33] C. V. Dang and G. L. Semenza, "Oncogenic alterations of metabolism," Trends in Biochemical Sciences, vol. 24, no. 2, pp. 68-72, 1999.

[34] L. M. Postovit, M. A. Adams, G. E. Lash, J. P. Heaton, and C. H. Graham, "Oxygen-mediated regulation of tumor cell invasiveness-involvement of a nitric oxide signaling pathway," Journal of Biological Chemistry, vol. 277, no. 38, pp. 35730-35737, 2002.

[35] J. Bussink, J. H. A. M. Kaanders, and A. J. Van Der Kogel, "Tumor hypoxia at the micro-regional level: clinical relevance and predictive value of exogenous and endogenous hypoxic cell markers," Radiotherapy and Oncology, vol. 67, no. 1, pp. 3-15, 2003.

[36] R. A. Gatenby and R. J. Gillies, "Why do cancers have high aerobic glycolysis?" Nature Reviews Cancer, vol. 4, no. 11, pp. 891-899, 2004.

[37] M. C. Brahimi-Horn and J. Pouysségur, "Oxygen, a source of life and stress,” FEBS Letters, vol. 581, no. 19, pp. 3582-3591, 2007.
[38] P. P. Hsu and D. M. Sabatini, "Cancer cell metabolism: warburg and beyond," Cell, vol. 134, no. 5, pp. 703-707, 2008.

[39] B. S. Peskin and M. J. Carter, "Chronic cellular hypoxia as the prime cause of cancer: what is the de-oxygenating role of adulterated and improper ratios of polyunsaturated fatty acids when incorporated into cell membranes?" Medical Hypotheses, vol. 70, no. 2, pp. 298-304, 2008.

[40] R. Moreno-Sánchez, S. Rodríguez-Enríquez, A. Marín-Hernández, and E. Saavedra, "Energy metabolism in tumor cells," FEBS Journal, vol. 274, no. 6, pp. 1393-1418, 2007.

[41] R. J. DeBerardinis, J. J. Lum, G. Hatzivassiliou, and C. B. Thompson, "The biology of cancer: metabolic reprogramming fuels cell growth and proliferation," Cell Metabolism, vol. 7, no. 1, pp. 11-20, 2008.

[42] Y. Chen, R. Cairns, I. Papandreou, A. Koong, and N. C. Denko, "Oxygen consumption can regulate the growth of tumors, a new perspective on the Warburg effect," PLoS One, vol. 4, no. 9, Article ID e7033, 2009.

[43] P. R. Stauffer and S. N. Goldberg, "Introduction: thermal ablation therapy," International Journal of Hyperthermia, vol. 20, no. 7, pp. 671-677, 2004.

[44] A. Chicheł, J. Skowronek, M. Kubaszewska, and M. Kanikowski, "Hyperthermia-Description of a method and a review of clinical applications," Reports of Practical Oncology and Radiotherapy, vol. 12, no. 5, pp. 267-275, 2007.

[45] J. R. Oleson, M. W. Dewhirst, J. M. Harrelson, K. A. Leopold, T. V. Samulski, and C. Y. Tso, "Tumor temperature distributions predict hyperthermia effect," International Journal of Radiation Oncology Biology Physics, vol. 16, no. 3, pp. 559-570, 1989.

[46] J. R. Lepock, "Cellular effects of hyperthermia: relevance to the minimum dose for thermal damage," International Journal of Hyperthermia, vol. 19, no. 3, pp. 252-266, 2003.

[47] M. Na, C. Chao, Z. Ai-li et al., "Thermal environmental effect on breast tumor growth," Journal of Shanghai Jiaotong University, vol. 27, no. 5, pp. 501-505, 2009.

[48] J. L. R. Roti, H. H. Kampinga, R. S. Malyapa et al., "Nuclear matrix as a target for hyperthermic killing of cancer cells," Cell Stress and Chaperones, vol. 3, no. 4, pp. 245-255, 1998.

[49] J. Dong, P. Liu, and L. X. Xu, "Immunologic response induced by synergistic effect of alternating cooling and heating of breast cancer," International Journal of Hyperthermia, vol. 25, no. 1, pp. 25-33, 2009.

[50] C. W. Song, "Effect of local hyperthermia on blood flow and microenvironment: a review," Cancer Research, vol. 44, no. 10, supplement, pp. S4721-S4730, 1984.

[51] C. W. Song, A. Lokshina, and J. G. Rhee, "Implication of blood flow in hyperthermic treatment of tumors," IEEE Transactions on Biomedical Engineering, vol. 31, no. 1, pp. 9-16, 1984.

[52] H. Maeda, J. Wu, T. Sawa, Y. Matsumura, and K. Hori, “Tumor vascular permeability and the EPR effect in macromolecular therapeutics: a review," Journal of Controlled Release, vol. 65, no. 1-2, pp. 271-284, 2000.

[53] D. T. Connolly, D. M. Heuvelman, R. Nelson et al., "Tumor vascular permeability factor stimulates endothelial cell growth and angiogenesis," Journal of Clinical Investigation, vol. 84, no. 5, pp. 1470-1478, 1989.

[54] Y. Shen, A. Zhang, and L. X. Xu, "Mechanical study on tumor microvessel damage induced by alternate cooling and heating treatment," in Proceedings of the 11th ASME Summer Bioengineering Conference (SBC'09), part A and B, pp. 593-594, June 2009. 
[55] J. P. Freyer and R. M. Sutherland, "Regulation of growth saturation and development of necrosis in EMT6/Ro multicellular spheroids by the glucose and oxygen supply," Cancer Research, vol. 46, no. 7, pp. 3504-3512, 1986. 


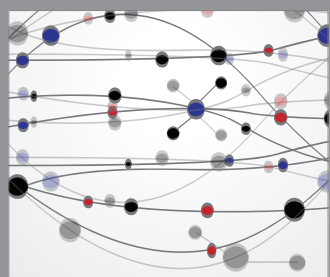

The Scientific World Journal
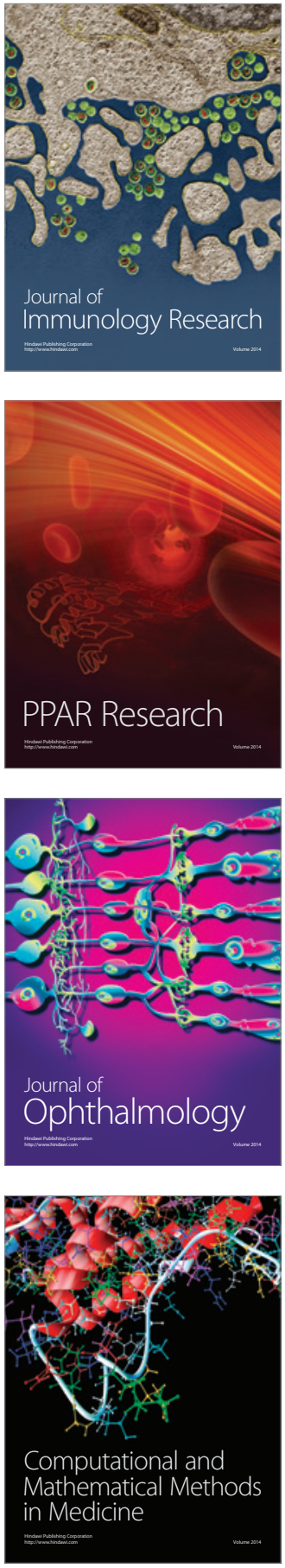

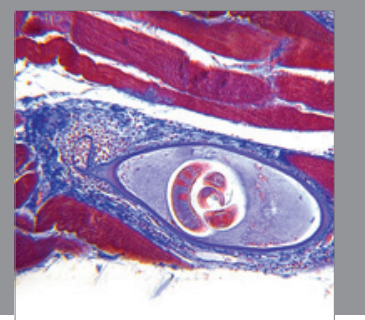

Gastroenterology

Research and Practice
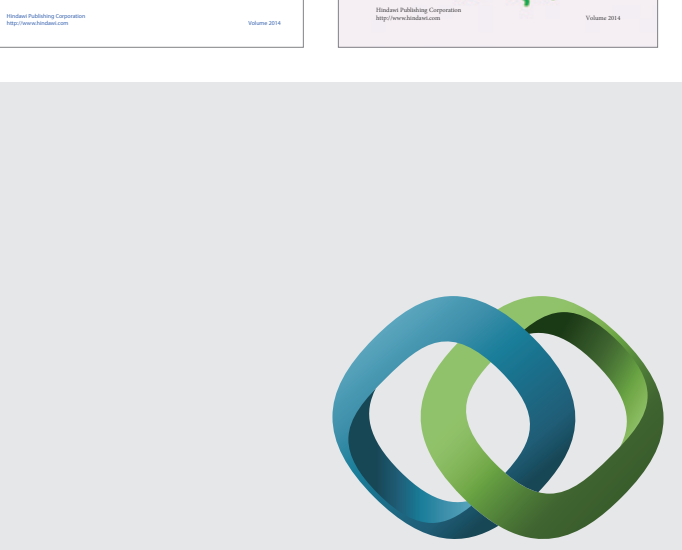

\section{Hindawi}

Submit your manuscripts at

http://www.hindawi.com
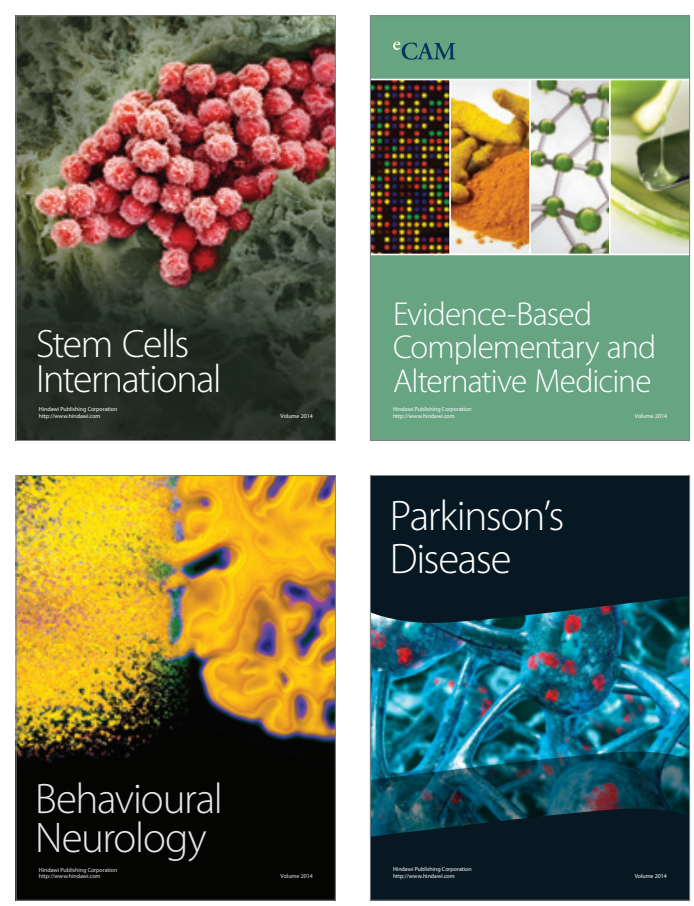

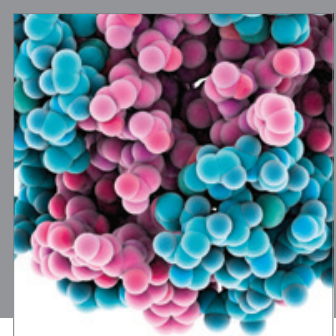

Journal of
Diabetes Research

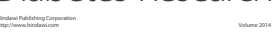

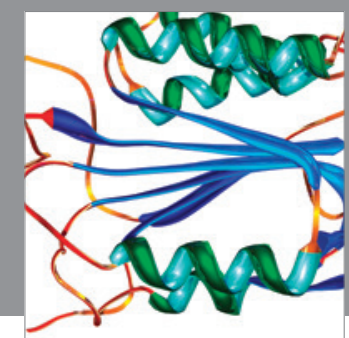

Disease Markers
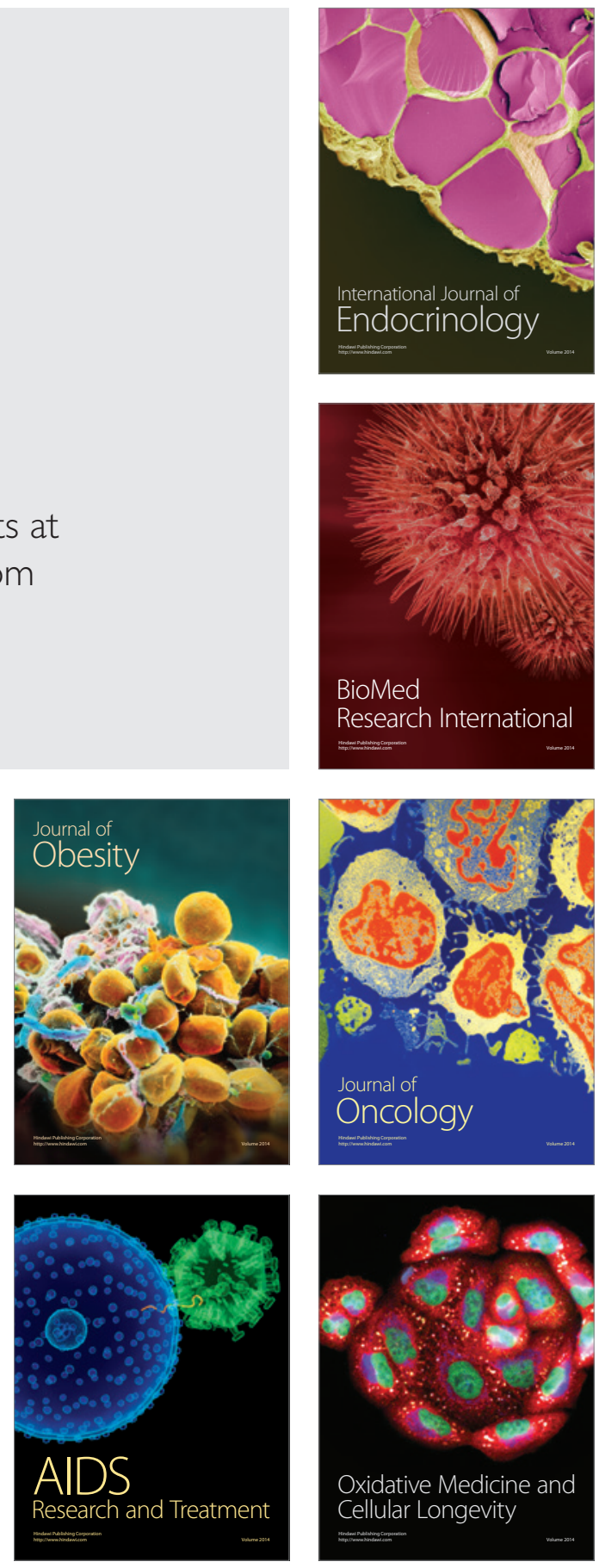